a pure cultivation of a bacillus. If a tube of gelatine peptone broth is inoculated, a series of convolutions form on the surface, with filaments growing down into the gelatine, or spreading out in somewhat parallel lines from the needle track. A very characteristic appearance is also obtained by growing it upon an oblique surface of agar-agar, which has suggested to me the name of "feather-bacillus" (Fig. 2). More beautiful preparations are made by employing a method which may be used in either plate or potato cultures. It is a method which enables us in many cases to study the relative position of individual micro-organisms one to another in their growth on solid cultivating media,

FIG. 1.

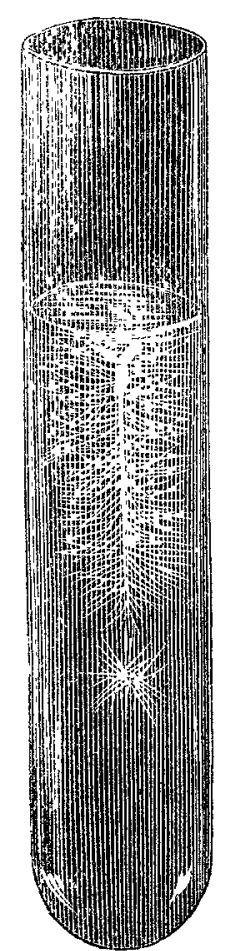

Pure cultivation of bacillus anthracis in gelatine peptone broth.

and is as follows: A perfectly clean, usually small-sized coverglass is carefully deposited on a plate or potato culture, and gently and evenly pressed down. One edge is then levered up carefully with a needle, and the cover-glass lifted off with forceps. It is then allowed to dry, passed through the flame, stained with a drop of fuchsin, the surplus stain washed off, and the specimen examined in water. To preserve it permanently, it is floated off the slide

with water, dried, and mounted in Canada balsam. In the case of plate cultures especially, as in this case, where no liquefaction has taken place, the growth or colony is bodily transferred to the cover-glass and a vacant space mapped out on the gelatine or agar-agar corresponding exactly with the form and size of the coverglass employed. In the case of the feather-bacillus, the individual bacilli are arranged in rows forming parallel lines, which at intervals become twisted up in the most exquisite convolutions, from which processes like the runners of a strawberry plant are sent off in various directions. These processes in turn become twisted up in varying shapes and forms.

Dresden.

City-road Hospital for Diseases of the Cimest.This hospital, which has been in course of enlargement for some time past by the erection of a new wing, is now approaching completion externally, but $£ 5000 \mathrm{i}$ still required before the building can be internally finished. The ground and first and second floors each contain three wards, $31 \mathrm{ft}$. by $18 \mathrm{ft}$., the third and dormer floors being set apart as dormitories for the matron and servants. The builder's contract amounts to close upon $£ 14,000$.

\section{THE MENTAL SYMPTOMS OF AORTIC REGURGITATION.}

WITH A SUMMARY OF THE NOTES ON FOURTEEN CASES,

BY J. HARRINGTON DOUTY,

SENIOR ASSISTANT MEDICAL OFFICER TO THE WORCESTER COUNTY ASYLUM.

A VERY large collection of observations, clinical and pathological, must yet be forthcoming before anything like a satisfactory conclusion can be arrived at with regard to the relations between heart lesions and certain forms o? insanity; the following notes, therefore, though perharg of small intrinsic value, are here recorded as an item towaris an ultimate development of the subject. During an examination of the hearts of a number of patients in the Worcester Asylum, I have so far met with fourteen in which there is incompetence of the aortic valves. I will give a brief account of them, and afterwards refer rather more in detail to a few of the most interesting.

CASE 1._-T.P_. Dementia and general paralysis; aotic regurgitation. Cause of insanity unknown. No heredity. Passed through the ordinary stages of general paralysis.

CASE 2.-T. D - Royal Engineers pensioner. Given to drink. Had sunstroke in the East. No heredity. Chronic mania; heard voices speaking to him day and night. Died suddenly from failure of the aortic valves.

CASE 3.-H. B-. Female. Mania. No cause known. No heredity. Aortic regurgitation. Had at first exaltad delusions; is now demented, and has signs of generd paralysis.

CASE 4.-S. P-. Strong heredity to insanity. Acute mania; fourth attack. Aortic regurgitation. Heard voices elephants, lions roaring, and whistles constantly blowing in her head.

CASE 5.-P. C-. Acute mania; fourth attack, Cause unknown, excepting previous attacks. No heredity. Aorti regurgitation. Wildly excited and violent on admission Now demented.

CASE 6.--A. J-Chronic mania. Cause unknown, to heredity. Aortic regurgitation. Heard voices, thunder and railway whistles whilst all was quiet around her Died of heart disease.

CASE 7.-A. W-. Chronic mania, ending in dementia Cause unknown. No heredity. Aortic regurgitation verr marked. Delusions chiefly of a suspicious nature. Died of heart disease.

CASE 8.--J. J. E-C. Chronic mania and (?) genema paralysis. Cause unknown. Heredity doubtful. Has delusions, symptoms of general paralysis, and a loud double aortic bruit.

CASE 9.--H.P_- Chronic mania. Very strong heredity (second attack). Double aortic bruit. Exalted delusions and at times violent and excited.

CASE 10.-H.D - Chronic mania (thirty years), Cause unknown. Very marked aortic incompetence. Has man strange hallucinations; hears a bull bellowing and people talking to her, especially when she is recumbent; sets "visions."

CAss 11.-F. J__. Mania; first attack. Cause unknorn. No heredity. Double aortic bruit. Hears voices and sees ghosts, and is irritable and bad-tempered.

CAsE 12.-E.D D. Dementia. Cause of insanity said to be drink. Aortic regurgitation and greatly dilated left rentricle, which, with an attack of erysipelas, caused her death. Had occasional hallucinations of sight.

CASE 13.-J. W-. "Melancholia cum stupore." Callsi said to be a recent severe attack of acute rheumatism. Io heredity. Aortic regurgitation, which caused death.

CASE 14.-M. H-_. Chronic mania. Strong lereditr Hears voices constantly talking to her, and consequents believes that people are following her to injure her. Es: well-marked signs of aortic regurgitation.

N.B.-In referring to the "cause of insanity" in the abore notes, I quote the information received on the subject from the friends on admission and subsequent inquiries.

The notes on the following three cases, selected from the above, are given as typical of the mental condition mast commonly obtaining as symptomatic of aortic incompetentit CASE 2.-T. D-, aged fifty, married, pensioner (Rors Engineers), well educated. IIe is a strong, well-nourished mall. 
with prominent eyes and well-marked aortic regurgitation. Capillary pulsation is visible. Mentally he is suffering from a delusional mania of two years' duration. ILe believes he can see the Royal Engineers surrounding him on all sides, and that, not content with this, they are up in a balloon overhead firing at him with rifles, which he hears day and night. He also hears constantly voices speaking to him by telephones-what he calls "sounds of tones of voices." Wherever he is, these sounds follow him and cause him the utmost annoyance, at times leading him to lose command of his temper, and to become violent, abusive, and agitated with rage. One day, whilst $I$. was reasoning with him as to the impossibility of hearing these voices where no telephones existed, he spoke up sharply and angrily: "Now, look here, doctor, I am no fool; I am not going to be humbugged by you any longer; you know perfectly well you can hear these scoundrels yourself, and if you say you cannot, then I say you are one of two things-a liar or a madman." From this time forward until his death he always believed that I was mad because of my ignorance of the proximity of his foes. He died suddenly one morning on getting out of bed, eighteen months after admission. An autopsy was held, but decomposition was so far advanced that examination was unsatisfactory. The frontal lobes, especially on the right side, were remarkably atrophied. The aorta for two inches from its origin was calcified, thick, and rigid; its valves were also calcified, two of them being immovable, and the third almost so. The left ventricle was enormously hypertrophied.

CASE 6.-A.J-, female, aged seventy-seven. Cause of insanity stated by certifier to be unknown. She has wellmarked diastolic bruit down the sternum, evidently of aortic origin; her pulse is collapsing, and no second sound is audible in the carotids; pulse visible in the capillaries. Mentally she is suffering from chronic delusional mania, her delusions mostly taking the form of hallucinations. She imagines she hears voices which she avers no one else can hear; these voices she thinks come from persons who are bent on drowning her in a cauldron of boiling water, the bubbling of which she also hears. Any unusual sound causes an illusion-e.g. a wheeibarrow wheeled down the corridor near her ward by a workman caused her to become greatly distressed and agitated on account of a terrible thunderstorm which she was sure was breaking overhead. The sound of water running from a tap into a bucket was to her the splashing of a huge waterfall. These hallucinations were very vivid. She would often say to me, "Don't say you do not hear them. You must hear them, unless you are stone deaf. Hark how they shout, and wrangle, and whistle. I can't sleep or rest for them; they will be the death of me if you cannot stop them." The old woman died four months after admission.

CASE 10. H. D - - female, aged fifty-six; chronic mania of thirty-nine years' duration. She has aortic regurgitation. For many years past she has had hallucinations of hearing and sight. She hears a bull almost nightly, which bellows and frightens her, amusing itself the while by routing up red currant trees and making holes in the ground. She hears rarious other queer sounds, and sees many curious sights. Her hallucinations are best marked, as is often the case, when she is lying down. Her temper is most irritable. A slight thing causes her to be greatly enraged. If questioned closely about her delusions, she abuses lier questioner loudly in the foulest language, threatens to kill him, and invokes the arch-demon to aid her in procuring his eternal destruction. About three months ago she had an embolism of a branch of the left middle cerebral artery, with aphasia and right hemiplegia. The paralysis has almost disappeared, but symptoms of secondary sclerosis are developing.

on reference to the above cases, we find that of the fourteen no less than eleven are cases of mania; there is one of dementia, one of dementia with general paralysis, and one of melancholia. It is evident, therefore, that as far as this small collection of cases is concerned, no less than 785 per cent. are instances of the association of mania with aortic regurgitation. Of these eleven cases of mania no less than seven possessed very marked auditory and visual hallucinations. It seems probable that when fuller statistics are forthcoming upon this subject we shall arrive at a conclusion that the typical mental symptom of aortic regurgitation is a delusional mania, coupled with a condition of extreme instability of temperament. Patients whose aortic ralves are incompetent, who are insane, are almost without exception of a touchy and excitable nature; very little upsets them; on the least provocation they will try to fight and struggle with their nurses to attain their own way, and are consecquently very difficult and dangerous cases to nurse. As surely as one discovers an aortic regurgitant bruit, almost so surely is one told by the attendant of the obstinacy and irritable temper of the patient, the least thing causing a violent outburst of anger and abuse. Another very common and interesting accompaniment of this valvular lesion is the prevalence of hallucinations, whose cause, however, it is not difficult to find. General clinical observation tells us how any alteration in the state of the circulation may cause symptoms which, if persistent, would soon develop into delusions. The tinnitus resulting from an anæmic or congested brain; the clouds and obscurities complained of by the subject of grave heart lesions; the giddiness and buzzing complained of by the anæmic girl; the troublesome throbbing, humming, or whistling noises which are heard by a patient who suffers from nocturnal palpitation, are familiar points of interest. It is probable that the hallucinations resulting from aortic: disease or any cause other than a local lesion of the ear or eye, are usually of central rather than of peripheral origin, for they are commou in cases in which the organs of special sense have been disorganised for years. We know that usually the flow of blood through the capillaries should be constant and equable. We may infer, therefore, that the blood flowing through the minute vessels of the internal ear, the retina, and the brain itself, does so in a fairly continuous stream in health; and that not only is the rate equable, but the pressure is also constant, or at any rate not subject to any sudden and frequent variations. Given, however, a grave reflux through aortic valves, and the converse of these conditions is established; the pressure in the capillaries becomes rhythmically various, increasing and diminishing with every cardiac systole and diastole. We know how acutely sensitive are the nervous centres and their peripheral arrangements of any departure from the normal. Such a condition of things, therefore, as that resulting from a grave aortic incompetence may produce a material interference with and perversion of the performance of their functions. It seems but natural, therefore, that, in the majority of cases associated with aortic reflux, hallucinations should be so common. Of course many things may occur to prevent the development of hallucinations, and complications may arise to mask the evidence of their existence. They are prominent symptoms in 50 per cent of the cases 1 have quoted. It is further noticeable that amongst the cases of mania several who had not hallucinations had exalted delusions and symptoms of general paralysis; this association of exaltation of idea and general paralysis with aortic regurgitation may be accidental, or it may result from the cardiac hypertrophy which naturally attends such lesions, and which has been described by Dr. Savage as productive of symptoms resembling those of general paralysis. One only of the fourteen cases was a simple dementia. The patient was listless, apathetic, and weak-minded, and had a feeble memory. Dr. Douglas Powell says he considers a certain amount of torpor and fatigue of mind to accompany many cases of aortic regurgitation, and to be relieved by the recumbent posture. This patient with dementia had, 1 should mention, hallucinations of sight, and would insist that she saw people walking about in the night She assisted one of the night nurses, and one night declared she saw me go into one of the wards at two or three in the morning, calling the nurse's attention to the "Doctor going down the passage." The case of "melancholia cum stupore" was the direct result of acute rheumatism, from which the patient had just recovered before admission, and which left him emaciated, feeble, and anæmic ; consequently the melancholic prevailed over the maniacal symptoms, which might have been prominent had his case been an uncomplicated one. It is interesting to note that heredity to insanity only existed in three out of the fourteen cases; sunstroke and drink were possible factors in two; in all the others no probable cause existed other than the heart lesion; and in the three with heredity one cannot say how far the heart disease was an exciting factor which developed the predisposing cause. How far the drink and sunstroke acted as causes in the two cases is also questionable; they neither of them recovered; one died, and the other is a chronic case. Had drink alone been answerable for the mania they might have recovered; atheroma and aortic disease, however, resulted from the drink, and hence death and chronicity. The typical form of insanity, then, resulting from aortic re- 
gurgitation is probably a delusional mania, frequently associated with very marked hallucinations and with a tendency to violent outbursts of rage and anger. That in many such cases no exciting cause for the insanity can be found other than the heart lesion, is also evident from the fact that in twelve out of only fourteen cases this was so. The recovery rate for these fourteen cases of insanity is 0 per cent.; this fact points strongly to the importance of the heart mischief as a causative factor; the insanity is a symptom of an incurable organic lesion, and as such is also itself incurable. Rest as a therapeutical agent is more or less serviceable in these cases; a few have recovered for a brief period sufficiently to leave the asylum, but they soon returned. If we had access to their aortic valves, and could repair their lesion, then we might possibly alter our prognosis.

Powick, Worcester.

\section{LYMPHATIC INFILTRATION OF THE SKIN IN CARCINOMA OF THE BREAST.}

\section{BY JOHN POLAND, F.R.C.S.}

IT is well known that there is a peculiar condition of the skin orer the breast which is clearly recognised as occurring in acute cancer of the breast and as cancerous infection yet its true nature and value appear to be but little understood. The condition I refer to is the brawnylike state-the early stage of the cuirass condition of French authors - a state in which more or less of the whole skin covering the mammary gland is rigid, cedematouslooking, and brawny, not unlike the rind of a lemon or boiled orange. This brawny condition of skin is found only in rapidly-growing carcinomatous tumours, or in those of slower growth where fresh increase has suddenly taken place. Later on the skin in the neighbourhood of the breast becomes in like manner affected, and on slight pressure with the fingers small local shot-like thickenings can be felt, which rapidly form into nodules and may present the cuirass condition above mentioned. The lymphatic glands in the axilla and above the clavicle may not be perceptibly enlarged, therefore we find it to be of especial importance that this condition of skin be understood in its true light, for undoubtedly all parts of the lymphatic system are already extensively infected by cancer cells, not only the network of lymphatics of the skin (as indicated by this condition), but also the subeutaneous lymphatics and the neighbouring glands. The decision of the surgeon as to the curability of the case by operation will consequently be much influenced by this sign. If he decide to operate under this condition, it will compel him to remove the skin very widely indeed, together with the pectoral muscle, and to clean out the axilla as thoroughly as possible, even though the glands be not felt by means of the fingers before operation. If a section be made through the skin and tumour, the former will be found to be enormously thickenedsometimes to as much as half or three-quarters of an inch,-white, and odematous, a little clear serum often running out on section. Extending outwards to this from the carcinomatous mass in the breast-substance beneath may be seen bands or lines of cancerous growth. In all the cases mentioned below the growth of the disease was central and had already invaded the nipple and part of the areola, and the skin over the whole area of the breast to a distance of four or five inches outwards from the nipple in this thickened condition, being at the peripheral parts thinner and less involved. Microscopically the dense network of cutaneous lymphatics, as has been ably worked out and described by Dr. Hoggan in the Pathological Society's 'Transactions for 1878, and in the Archives de Physiologie for 1880, will be found to be plugged with cancer cells, which, later on spreading to the walls of the lymphatics, develop secondary nodules. The tissue of the skin itself is altered much in character, becoming hard, compact, and more hulky. In the course of a few months at Guy's Hospital I observed three such cases, and a fourth closely resembling these in its clinical features and naked-eye appearances on section, but which on microscopical examination proved to be an entirely inflammatory condition of the skin over a breast affected with acute cancer, and due to the repeated application of leeches and poultices to the cancerous breast. The erroneous supposition that lymphatic infilts tion of skin existed in this case might have been arnidhi? had the extreme tenderness and slight red blush (which ar: not present in the cases we are considering) been taben into account. Unfortunately, in one only of the three cass was a careful microscopical examination made of the skin In all, however, the rough appearances were the same, an in all there were numerous minute cancerous nodules inil. trating widely the pectoral muscles in rows like. strings of beads parallel with the muscular fibres and running into eact other, indicating that the cancer cells were at this tim widely disseminated. Death occurred in all the cases a fers weeks after removal of the breast, and in two of the cass the affected axillary glands.

The case from which the microscopical sections wen made was that of Susan $\mathrm{F}$ into Guy's Hospital on September 6th, 1883. She hact had ten children, nine of whom she had suckled chiefly with the left breast. Thirteen months before she felt acute pain and a "lump" on the outer side of the right breast. This ver gradually increased for twelve months, when it was of the size of a five-shilling piece. During the last three month it had rapidly increased in size, with pain and swellingi the right axilla. Three weeks before admission the skin oret the breast had begun to assume the condition described belor, On admission the site of the right breast was occupied br hard rounded mass, six by four inches, implicating the sin but freely movable over the pectoral muscle. The nipple was retracted and the areola puckered and irregular wit mall nodules. The skin covering the whole of the mammen gland had an oedematous and irregular appearance, lookin and feeling not unlike boiled orange-rind, and for a distan of three inches, more especially marked abore the nipple was exceedingly brawny and studded with numerous sill elevations about the size of millet-seeds. Some glands und the anterior fold of the axilla were felt to be enlarged, o Sept. 14th the breast was removed, together with a larg amount of thickened skin. Four glands were turned out the axilla and a portion of the pectoral muscle cutars The latter was found to be so largely scattered with smil cancerous nodules that the operation was not proceeded mil any further. Recurrence and death rapidly took place, section, the growth was central, white, tough, and typical acute scirrhus of the breast, with trabeculæ of growther tending to the skin. The nipple was invaded by the grow The skin external to the nipple was three-quarters of an inch thick, elsewhere about one-eighth of an inch, gradual diminishing in thickness towards the periphery of the breat Specimens of the skin radiating from the nipple outwark were sent to Dr. Hoggan, together with similar portions i" the skin from the case with supposed lymphatic affection. Dr. Hoggan kindly made sections of the skin in each of th two cases, and sent me the following report of the result ti his examination:-

"I have now completed the preparation of the portions of skin you left with me, and carefully examined them gat compared them with each other. Your two cases of canet. which you say were clinically almost identical, turn out be entirely dissimilar when examined histologically. Toit first case was typical of cancer spreading by means of tip lymphatics, even in the peripheral direction-that is to sor. in the direction opposite to that of the lymphatic gands Indeed, in the unfortunately too small specimen of 8 sin from the case which you gave me, the only locality mher? cancer cells were to be seen in the whole tissue was wititin the lympatic vessels of the skin. Only, however, the defort lymphatics of the skin were plugged with cancer cellsint th' specimen, the most superficial lymphatics being onlypluge: with lymph cells, into which the cancerous infection had an as yet spread. Even of the deeper lymphatics the walls bit. not as yet become cancerous, and consequently there Fi: $^{2}$ formation or development of secondary cancerous tumols: from the cancerous lymphatics. Altogether, your firt was identical with the one I published, jointly with wife, in the Archives de Physiologie for 1880; the questite of comparative plugging of the lymphatics or derent ment of secondary tumours being dependent on the st: to which the disease had reached at the exact spot if th which your specimen had been taken. As in my case presence of the cancer cells, even within the lympledtly had modified the character of the gelatinous tissue of skin, which was compact, and afforded no evidence of eitt: the presence of oedema or of cell infiltration. Your seeth case, on the contrary, showed no plugging of the lymplas 